Congreso Internacional de Gestión Educativa, 06 y 07 de noviembre de 2020.

Pontificia Universidad Católica del Perú

\title{
Prácticas Inclusivas Durante El Covid 19; Una Mirada Desde La Gestión Comunitaria Y Académica
}

\author{
María Cristina Olarte Artunduaga \\ Universidad Pedagógica y Tecnológica de Colombia UPTC \\ maria.olarte@uptc.edu.co \\ https://orcid.org/0000-0002-3826-5704
}

\section{Resumen:}

La ponencia titulada "PRÁCTICAS INCLUSIVAS DURANTE EL COVID 19 UNA MIRADA DESDE LA GESTIÓN COMUNITARIA Y ACADÉMICA" tiene como objetivo reconocer en las prácticas pedagógicas los procesos de inclusión en los niños y niñas de básica primaria durante la cuarentena por el COVID-19 en la Institución Educativa Técnico San Martin De Tours, a través, de la reconstrucción de una experiencia vivida que dé espacio al diálogo, al debate y a la reflexión crítica, de tal manera que produzca una nueva mirada sobre la práctica pedagógica, en un tiempo en que emerge la necesidad de un cambio en las dinámicas del currículo; cuando el hogar se convierte en un espacio educativo y el docente debe diseñar diferentes estrategias de enseñanza, enfocadas a la atención de población diversa. Para empezar, se expone la práctica inclusiva, desde la diversidad y la diferencia con autores contemporáneos como: Corcini (2018), Skliar (2005) y la visión de Yarza (2008) desde el punto de vista de los dispositivos de formación, en un contexto en donde la inclusión educativa plantea retos a los procesos formativos de educadores, preguntándose por la importancia del aprender juntos o el aprender separados. De igual manera, se revisa el objetivo de la gestión educativa en lo que se ha construido como concepto de escuela, haciendo énfasis en la importancia de las condiciones y capacidades de la organización del sistema escolar, que hagan posible la inclusión y la diversidad, a través de políticas y programas diseñados con el fin de garantizar equidad y respeto por la diferencia. Para finalizar, se establece un análisis entorno a la articulación de la práctica pedagógica y la gestión del sistema frente a la pandemia global que nos ha sacudido, y que obliga a la educación a dirigirse hacia un cambió experimental en este tiempo de COVID-19.

Palabras clave: Práctica Inclusiva, Diversidad, Diferencia, Gestión Educativa, Covid19

\begin{abstract}
:
The presentation entitled "Inclusive Practices During Covid 19 A View From The Community And Academic Management" aims to recognize in pedagogical practices the inclusion processes in elementary school boys and girls during the quarantine by COVID-19 in the Institution Educational Technical San Martin De Tours, through the reconstruction of a lived experience that gives space for dialogue, debate and critical reflection, in such a way that it produces a new look at pedagogical practice, at a time when the need for a change in the dynamics of the curriculum; when the home becomes an educational space and the teacher must design different teaching strategies, focused on serving a diverse population. To begin with, inclusive practice is exposed, from the diversity and difference with contemporary authors such as: Corcini (2018), Skliar
\end{abstract}


Congreso Internacional de Gestión Educativa, 06 y 07 de noviembre de 2020.

Pontificia Universidad Católica del Perú

(2005) and Yarza's (2008) vision from the point of view of training devices, in a context in which educational inclusion poses challenges to the formative processes of educators, wondering about the importance of learning together or learning separately. In the same way, the objective of educational management is reviewed in what has been built as a school concept, emphasizing the importance of the conditions and capacities of the organization of the school system, which make inclusion and diversity possible, to through policies and programs designed to ensure equity and respect for difference. Finally, an analysis is established around the articulation of pedagogical practice and the management of the system in the face of the global pandemic that has shaken us, and that forces education to move towards an experimental change in this time of COVID19.

Keywords: Inclusive Practice, Diversity, Difference, Educational Management, Covid19

\section{Introducción}

En la actualidad la educación enfrenta el reto constituido por la diversidad, como consecuencia de que asistimos a un momento histórico caracterizado por las nuevas dinámicas sociales, situación que se refleja en las instituciones educativas. El trabajo que presentamos en este documento, trata sobre la diversidad escolar desde una visión conceptual y práctica frente al tema en un escenario incierto, complejo y cambiante donde tienen lugar interacciones que difícilmente pueden preverse y que son permeadas por las políticas, lineamientos y situaciones propias del entorno.

El objetivo principal de esta investigación es analizar prácticas educativas teniendo como como línea base la validación de la sistematización de experiencias en el proceso pedagógico e interdisciplinar de la práctica pedagógica inclusiva en época del Covid-19, por parte del profesorado, los directivos docentes y el equipo de apoyo en las aulas de básica primaria de la Institución Educativa Técnico San Martin De Tours, a través, de la reconstrucción de una experiencia vivida que dé espacio al diálogo, al debate y a la reflexión crítica, de tal manera que produzca una nueva mirada sobre el quehacer pedagógico, en un tiempo en que emerge la necesidad de un cambio en las dinámicas del proceso de enseñanza y aprendizaje; cuando el hogar se convierte en un espacio educativo y el docente debe diseñar diferentes estrategias de enseñanza, enfocadas a la atención de población diversa.

\section{Definición del objeto o problema de estudio}

Al hablar de educación inclusiva, implica hablar de discapacidad, en palabras de Escallón (2013)"Se reconoce que la educación inclusiva por diversos motivos ha estado inmensamente ligada a la educación de la población con discapacidad y esto permite explicar muchos de los obstáculos que enfrenta en la actualidad" (p. 29), por tal razón, la educación inclusiva ha sido vista como un proceso exclusivo para niños con alguna discapacidad física, psíquica, cognitiva, sensorial, etiquetando un grupo en particular, a partir de las carencias, olvidando las potencialidades y habilidades.

En la Institución Educativa Técnico San Martin De Tours, al hablar de inclusión se hace referencia a la práctica pedagógica con poblaciones heterogéneas que presentan

Sistematización de la práctica pedagógica inclusiva en época del Covid-19 - Olarte Artunduaga, María Cristina

DOI: https://doi.org/10.18800/cige2020.006 
Congreso Internacional de Gestión Educativa, 06 y 07 de noviembre de 2020.

Pontificia Universidad Católica del Perú

condiciones de aprendizaje diversas, siendo este, un reto que requiere dinamismo, para lograr acoger a toda la población brindando respuestas educativas, pedagógicas y didácticas que permitan el aprendizaje y fomenten mecanismos de participación de los niños y niñas, en un currículo flexible y acorde a la atención de todos y todas.

A comienzos del año 2020 a nivel mundial se identifica un virus de propagación masiva que lleva a vivir condiciones de aislamiento obligatorio para toda la población, convirtiendo al hogar en el colegio, y a los maestros en aprendices virtuales que intentan apropiarse de estrategias metodológicas, técnicas, didácticas, con el fin de transmitir contenidos a la población estudiantil. El MEN, ante la situación presentada definió lineamientos y orientaciones para atender a los niños y niñas durante el trabajo que se denominó "Servicio de educación en casa", la cual, consta de unas directivas ministeriales que son emitidas de acuerdo con las necesidades que se van presentando según el comportamiento del virus.

Durante el segundo trimestre del año 2020 los niños y jóvenes del mundo se han visto afectados al tener que hacer frente a la pandemia global que nos ha sacudido, en este sentido, la educación tomó un cambio experimental hacía lo digital, aunque ya existe la educación en línea, en este tiempo de COVID-19 se ha reforzado aún más. El rol del estudiante y del profesor se une, ambos deben trabajar en equipo para reforzar el aprendizaje y para saber cómo enseñar y aprender. Es así, como las plataformas y aplicaciones digitales son parte de las estrategias para facilitar la comunicación. Aunque para algunos, resulta ser una complicación incómoda, para otros, la situación es aún más preocupante. En instituciones educativas donde el $70 \%$ de los estudiantes vienen de familias de bajos ingresos, llevar la escuela a casa significa descartar la educación como prioridad por falta de acceso a la tecnología o conectividad.

Como resultado, de la situación presentada se da inicio a la reflexión sobre las prácticas inclusivas, durante la cuarentena por el COVID-19 y de igual manera se pretende hacer visibles los saberes y significados que los diferentes actores: niños, niñas, padres de familia y principalmente los maestros de la institución han experimentado y la manera como se han desarrollado, con el fin de resaltar el sentido y la significación de la práctica pedagógica en un proceso que además de ser inclusivo, permita reflexionar sobre el que hacer educativo en momentos, donde el tiempo y el espacio exige acciones educativas diferentes.

De esta manera, nos lleva a preguntarnos ¿Qué tipo de experiencia ha provocado el distanciamiento social obligatorio?, por ejemplo, la comunicación se ha complejizado dada la distancia, los nuevos canales de comunicación que siempre deben tener un intermediario entre maestro y estudiante: en el caso de los niños sordos se requiere un intérprete que comunica al niño pero se interrumpe la relación con el docente, para los niños ciegos ha resultado difícil conectarse por el sistema virtual, para los niños denominados con discapacidad cognitiva los intermediarios son los padres que muchas veces no cuentan con herramientas pedagógicas y algunos de ellos no han desarrollado un lenguaje escrito.

Por otra parte, es necesario reconocer que los espacios y materiales con que cuenta el espacio presencial del aula es determinante en cuanto a contacto con objetos, formas, texturas, material especializado para atender cada discapacidad, siendo esta una necesidad que, como institución, tiene la responsabilidad de atender, con el fin, de conseguir el desarrollo integral de los alumnos y alumnas. Por ello, se convierte esta

Sistematización de la práctica pedagógica inclusiva en época del Covid-19 - Olarte Artunduaga, María Cristina

DOI: https://doi.org/10.18800/cige2020.006 
Congreso Internacional de Gestión Educativa, 06 y 07 de noviembre de 2020.

Pontificia Universidad Católica del Perú

situación en una preocupación, que incide en la restructuración de las técnicas y herramientas adecuadas para conseguir el objetivo de los procesos de enseñanza, dentro de la situación actual.

Siguiendo a Gimeno (1995) "La constante en la búsqueda de lo que pudiéramos llamar un sistema educativo y una escuela progresista ha sido la de no separar, la de integrar las diferencias en aras de la igualdad." Expuesto lo anterior, emerge como pregunta de investigación ¿Cómo se ha desarrollado las prácticas inclusivas en los niños y niñas de básica primaria durante la cuarentena por el Covid-19?

\section{Fundamentación teórica}

El tema de la diversidad es complejo, está presente en los discursos académicos, culturales y es una prioridad en las políticas educativas como una forma de dar respuesta a los principios de equidad y calidad de la educación. Además, la diversidad se ha hecho cada vez más evidente, como también la brecha cultural entre los actores que intervienen en el sistema que conocemos como escuela. Esto ubica a la diversidad como campo temático en un espacio trascendental en la educación, donde, gracias a las políticas de cobertura y permanencia, ha aumentado la diversidad de alumnos que accede a ella, sin embargo, los sistemas educativos siguen respuestas homogéneas que no satisfacen las necesidades y situaciones individuales de los alumnos. Lo anterior se ve reflejado en los altos niveles de deserción y los bajos niveles de aprendizaje que afectan en gran medida a las poblaciones de mayor vulnerabilidad.

De tal manera que, en la educación siempre ha existido y se ha excluido de algunos conocimientos a personas por su sexo, edad, color o entorno social. Aunque el concepto de diferencia ha evolucionado hacia la idea de que niñas y niños deben tener oportunidades equitativas de aprendizaje independientemente de sus antecedentes sociales, culturales, de sus habilidades y capacidades. De ahí, que la inclusión educativa sea definida como la estrategia y política, que pretende asegurar la integración de niños y niñas en el sistema educativo, dando numerosas respuestas a los docentes, estudiantes y familias enfocadas hacia el respeto y la tolerancia frente a la diversidad y la diferencia,

En este sentido, la reflexión parte desde la concepción de la diversidad y de la diferencia, las políticas y la gestión educativa; componentes que permiten alinear las concepciones y acciones en función del logro de una práctica inclusiva efectiva más clara, y que haga frente al desafío que representa la tarea de educar a todos los niños y niñas dentro de un sistema educativo que responda a la diversidad de necesidades y que, a su vez, valore pedagógicamente la integración de todos y todas. En situaciones donde las dinámicas se movilizan ante una problemática mundial que suspendió las actividades de más de 1,215 millones de estudiantes de todos los niveles educativos, en el planeta entero (UNESCO. 2020).

Un primer aspecto, que se debe dar en torno a la reflexión acerca de la diversidad, es el carácter gubernamental y biopolítico de las políticas educativas inclusivas, que son orientadas por el estado y que permiten dar una mirada a los elementos económicos y políticos. Evitando tomar un posicionamiento a favor o en contra de las políticas y prácticas inclusivas; no se trata aquí, de hacer un juicio de valor sobre ellas, sino con la finalidad de realizar un análisis hacia la interpretación de las políticas educacionales de

Sistematización de la práctica pedagógica inclusiva en época del Covid-19 - Olarte Artunduaga, María Cristina

DOI: https://doi.org/10.18800/cige2020.006 
Congreso Internacional de Gestión Educativa, 06 y 07 de noviembre de 2020.

Pontificia Universidad Católica del Perú

inclusión en las escuelas desde la visión docente (Corcini, 2018) Se trata entonces, de examinar aquello que dicen las políticas inclusivas, buscando investigar los referentes teóricos y conceptuales que han servido de base para el reconocimiento de las diferencias y la apertura al concepto de inclusión.

Al abordar, la diversidad desde una mirada que se focaliza en la revisión de la complejidad que representan las metas de una realidad que cambia y que está inmersa en las perspectivas diversas sobre los propósitos de la educación, a la cual, se añaden los desafíos y oportunidades para todos y todas, dentro de lo que hoy conocemos como educación. Empleando las palabras de Skliar (2005):

Por eso creo que en educación no se trata de caracterizar mejor qué es la diversidad y quién la compone, sino en comprender mejor cómo las diferencias nos constituyen como humanos, cómo estamos hechos de diferencias. Y no para acabar con ellas, no para domesticarlas, sino para mantenerlas y sostenerlas en su más inquietante y perturbador misterio (pp.20).

Es decir, el autor expresa que las dinámicas sobre la normalización de lo anormal están dadas en el aula, olvidando lo importante de educar en la diversidad, y no diferenciándola, sino incluyéndola como una norma sobre la cual se puede y se deben movilizar los sujetos, proyectos de vida, e incluso ideas sociales, políticas o económicas.

Por su parte, Yarza (2008) indica que "El auge de las reformas (constitucionales) en que la diversidad da paso al reconocimiento de derechos jurídicos a "las minorías", se articula a nuevas prácticas (políticas y educativas) para la inclusión”. Dicho de otra manera, las reformas y políticas enfocadas hacia la diversidad y la diferencia, traen consigo programas y políticas educativas, que tiene como fin o pretensión dar a cada uno de los sujetos del sistema una fórmula que invita a la equidad y al acceso de la educación para todos y todas, sin olvidar los dispositivos de formación, en un contexto en donde la inclusión educativa está planteándole retos a los procesos formativos de educadores, preguntándose por la importancia de ¿Aprender juntos o aprender separados?

Así pues, la educación de calidad debe promover prácticas educativas inclusivas en donde la educación tenga principios de igualdad, equidad y justicia social, además, promuevan la inclusión social siendo esta una tendencia de las políticas y por consiguiente un factor relevante de la gestión educativa. La cual se define como el proceso de organización que está orientado hacia el mejoramiento continuo de la calidad educativa, el cual se ha logrado por medio del desarrollo de subprocesos como la caracterización, la planeación, la ejecución, el seguimiento, la evaluación y el reconocimiento de experiencias significativas en relación con el quehacer educativo y el fortalecimiento institucional. Todo dentro de un marco jurídico que lo regula y establece las directrices de ejecución, control y retroalimentación. Así mismo, dentro de las categorías de la gestión educativa como sistema se encuentran: la gestión institucional que hace referencia a la estructura, la gestión escolar desde la mirada comunitaria y la gestión pedagógica que se refiere a los procesos de aula, que son los diferentes ámbitos

Sistematización de la práctica pedagógica inclusiva en época del Covid-19 - Olarte Artunduaga, María Cristina

DOI: https://doi.org/10.18800/cige2020.006 
Congreso Internacional de Gestión Educativa, 06 y 07 de noviembre de 2020.

Pontificia Universidad Católica del Perú

y niveles en las que se divide la gestión en las que puede actuar y generar cambios de mejora educativa de acuerdo a las exigencias de la normatividad.

Teniendo en cuenta, que diversidad ha sido una palabra que ha ido evolucionando en base a la escuela que ha cambiado de modelos educativos, ahora los grupos son heterogéneos y se integran educandos con diferentes capacidades, esto nos ha llevado a que en las instituciones educativas se debe integrar la diversidad y convertirla en un contenido de aprendizaje más y, al mismo tiempo, deben propiciar la formación de sujetos con las suficientes capacidades y habilidades que le permitan desenvolverse en la diversa y compleja sociedad que le rodea. Como consecuencia, de estas nuevas visiones, las instituciones educativas se ven en la necesidad de renovar las fórmulas tradicionales de la gestión escolar, por otras que busquen respuestas contextualizadas a las interrogantes y las demandas que día tras día la sociedad presenta.

De igual manera, la gestión educativa ha construido un concepto de escuela, haciendo énfasis en la importancia de las condiciones y capacidades de la organización del sistema escolar que hacen posible la inclusión y la diversidad, a través de políticas y programas diseñados, con el fin de garantizar equidad y respeto por la diferencia. Es por esto que, la relación entre gestión y educación es notoria, según Cassasus (2000) el objetivo principal de esta disciplina es aplicar los principios de la gestión en la educación y afirma que esta disciplina no es solamente teórica, ya que, está influenciada por la cotidianidad de su práctica, es decir es una disciplina aplicada y un campo de acción.

Por su parte, Sander (2002), habla de la gestión educativa desde una perspectiva teórica, donde indica que la administración en la educación no es un hecho gratuito, por el contrario, es un proceso de construcción histórica en cual todos siguen participando y escribiendo al respecto. Por esta razón, las políticas han replanteado sus leyes y requerimientos educativos, en busca de la adecuación de mejores prácticas y teniendo en cuenta que cada persona es un sujeto de derechos y por ende se le debe garantizar los mismos; en todos los campos sociales y políticos.

En relación a este aspecto, valdría la pena analizar como la gestión académica enfrenta qué tan inclusivas son las prácticas, currículos y estrategias que se desarrollan dentro de la llamada educación inclusiva, razón por la cual, en los discursos sobre educación, se evidencia la 'preocupación por dar al concepto la participación y la fuerza necesaria, que implica "una responsabilidad recíproca" (Blak-Hawkins, y Rouse, 2007: 48-49). Que invita a revisar las políticas, y prácticas escolares haciendo énfasis, en la esencia misma de la educación inclusiva, evitando planteamientos que conviertan los saberes y significados acerca de la práctica de inclusión en un discurso que tienen como tópicos de estudio simplemente el aula y el sistema. Para Soto Calderón (2003) el paso previo para una clarificación del sentido de la inclusión educativa es "el análisis y la discusión de los diferentes procesos en que se ha enmarcado la experiencia educativa de las personas con necesidades educativas especiales; así como los procesos de formación de los docentes y otros profesionales relacionados con estas personas".

Lo cual significa, que la gestión educativa desde la perspectiva académica ha surgido como el motor que impulsa y enfrenta los cambios que emergen de situaciones dadas por el contexto, lográndolo a través de la reflexión que permite reconocer las problemáticas educativas, tanto en las políticas como las prácticas pedagógicas 
Congreso Internacional de Gestión Educativa, 06 y 07 de noviembre de 2020.

Pontificia Universidad Católica del Perú

presentes. Se refiere entonces, al diseño de una idea que permite la deliberación sobre los aprendizajes que garantizan la experiencia con población diversa.

Por otra parte, en un contexto como el actual que se ha visto afectado por la pandemia del COVID.19, donde cientos de miles de personas en todo el mundo han modificado sus dinámicas de vida, y se han visto obligados a unas condiciones de aislamiento obligatorio, convirtiendo al hogar en el colegio, y a los maestros en aprendices virtuales que intentan apropiarse de estrategias metodológicas, técnicas, didácticas, con el fin de transmitir contenidos a la población estudiantil. El MEN, ante la situación presentada define lineamientos y orientaciones para atender a los niños y niñas durante el trabajo que se denominó "Servicio de educación en casa", la cual, consta de unas directivas ministeriales que son emitidas de acuerdo con las necesidades que se van presentando según el comportamiento del virus.

El MEN diseña plataformas digitales que los docentes deben manejar con el propósito de flexibilizar los currículos, transversalizar asignaturas que antes no se consideraban importantes como la ética, la educación física, cátedra de la paz, manejo de emociones, todos estos ajustes sugeridos no hablan de las formas como se puede modificar y gestionar la academia desde una mirada comprensiva de la diversidad que está aún más presente. Siendo entonces este el reto de la gestión académica, donde la sinergia de la institución exige el diseño y aplicación de prácticas pedagógicas emergentes que requieren de la participación de los padres, acceso a nuevas tecnologías, movilidad del currículo y continuidad pedagógica.

En Colombia, las instituciones educativas cerraron a mediados de marzo cuando se definió suspender las clases presenciales en los colegios del país. A partir del lunes 16 de marzo, los niños, niñas y jóvenes de instituciones de educación pública y privada del país, no tendrían más clases presenciales para proteger la salud de todos. Posteriormente el MEN emite la circular 021 del 17 de marzo de 2020, donde da las orientaciones para el desarrollo de procesos de planeación pedagógica y trabajo académico en casa como medida para la prevención de la propagación del Coronavirus (COVID-19). Llevando a todas las entidades territoriales del país a definir los tiempos y las estrategias con que seguirán atendiendo a los estudiantes durante el aislamiento obligatorio.

Como resultado, de la situación presentada se da inicio a la reflexión sobre las prácticas inclusivas y la manera en que la comunidad debe desafiar las nuevas condiciones del sistema escolar, durante la cuarentena por el COVID-19, donde las pérdidas del espacio escolar hacen visible la reestructuración de los saberes y significados de los diferentes actores: niños, niñas, padres de familia y principalmente los maestros de la institución se encuentran experimentando en una escuela ausente y que ya no educa de una manera tradicional dentro del aula, que se ve enfrentada a una coyuntura donde los ejercicios propios de la escuela cambian el sentido y la significación de la práctica pedagógica en un proceso que además de ser inclusivo, permita reflexionar sobre el que hacer educativo en momentos, donde el tiempo y el espacio exige acciones educativas diferentes. Al llegar a este punto, la reflexión nos lleva a reconocer que con la llegada del COVID-19 no se detuvo la vida escolar, sino que por el contrario surgieron pedagogías emergentes y nuevas maneras de construir saberes y aprendizajes. De esta manera, la actividad escolar se modifica, pero no cambia su finalidad (Diaz, 2020). 
Congreso Internacional de Gestión Educativa, 06 y 07 de noviembre de 2020.

Pontificia Universidad Católica del Perú

Al respecto se puede concluir, que en el momento actual se ha evidenciado más la necesidad de revisar nuestro sistema educativo y alinearlo con la realidad en la que vivimos, donde el surgimiento de prácticas pedagógicas de carácter emergente lleva a la escuela a unas nuevas dinámicas donde intervienen factores internos y externos, que modifican al currículo y más aún cuando hace referencia a la práctica pedagógica con poblaciones heterogéneas que presentan condiciones de aprendizaje diversas, siendo este, un reto que requiere dinamismo, para lograr acoger a toda la población brindando respuestas educativas, pedagógicas y didácticas que permitan el aprendizaje y fomenten mecanismos de participación de los niños y niñas, en un currículo flexible y acorde a las necesidades del ambiente de aprendizaje, donde la diversidad y la diferencia están presente.

\section{Metodología}

Desde una perspectiva metodológica, se decide retomar el planteamiento de Jara (2013), respecto a los pasos que orientan cómo sistematizar, y las dos condiciones formuladas por éste para el desarrollo de una sistematización: las condiciones personales y las condiciones institucionales. Con el fin de generar una reflexión que conduzca a la reconstrucción del saber pedagógico, se determinó rescatar la experiencia personal de los docente de la sección primaria; posibilitando, las condiciones personales, a través del intercambio narrativo y de compartir el proceso práctico situado en el contexto; generando la reflexión, reinterpretación y reconstrucción del sentido de la propia práctica, partiendo de valorar las vivencias de estos docente como fuente de aprendizaje y de nuevos conocimientos, que aportan a la práctica pedagógica inclusiva.

Además, se rescata la importancia de las condiciones institucionales, para situar en esta sistematización como lugar de experiencia se reconoce el aporte y vínculo particular de las interacciones en el espacio; es decir, la experiencia del actor o actores que participan en el proceso.

Para el desarrollo de la sistematización se define la siguiente ruta

1. Punto de partida; momento que recoge las preguntas movilizadoras iniciales, y las motivaciones para definir el objeto de esta sistematización de experiencias.

2. ¿Qué sistematizar?: para esta sistematización, se define la indagación desde una experiencia personal de los docentes, que ha desempeñado este rol en un contexto escolar durante un periodo de tiempo de un año; donde se consideró determinante recoger sus percepciones y observar su ejercicio profesional en campo; procurando retomar, los aspectos más representativos de su quehacer diario.

3. ¿Para qué sistematizar?: para definir los objetivos, en relación a la pregunta de investigación y se precisan los aspectos centrales para su posterior interpretación y análisis. 
Congreso Internacional de Gestión Educativa, 06 y 07 de noviembre de 2020.

Pontificia Universidad Católica del Perú

4. Los caminos para la comprensión teórica: consiste en la revisión de un marco legal y el recorrido de posturas teóricas de autores e instituciones que retoman las categorías de análisis: Integración Educativa, Prácticas Inclusivas, Educación Inclusiva, Población Diversa

5. La reconstrucción de la experiencia compartida: aquí se realizará la organización y clasificación de la información más relevante, presentando la recuperación de las distintas observaciones a la práctica cotidiana del actor principal, sus percepciones en torno a su ejercicio profesional, y acciones pedagógicas que configuran su rol como docente, desde su propia voz, como eje principal de este proceso de sistematización.

6. La interpretación crítica: se realizará las reflexiones sobre las tensiones más importantes, los puntos en común entre los actores principales de este ejercicio de sistematización, y las confrontaciones que dan fruto a la reconstrucción de sentido, tanto de las nuevas miradas al rol del docente como a las exigencias que se pueden dilucidar de la Educación Especial en constante construcción.

7. El punto de llegada: se da respuesta a los objetivos planteados durante el proceso investigativo, dando lugar a la formulación de las conclusiones y proyecciones que surgieron a partir de las reflexiones y aprendizajes significativos obtenidos de la sistematización

\section{Resultados y Conclusiones}

A partir de la sistematización de la experiencia en la Institución Educativa Técnico San Martin De Tours, se tiene la posibilidad de:

1. Reevaluar y al mismo tiempo, reflexionar sobre el papel como docente en tiempos donde las dinámicas se han modificado.

2. Se evidencia que la sistematización fortalece el rol del docente, y permite reflejar de manera más particular y mucho más clara su quehacer.

3. A través del desarrollo de la investigación se puede llevar a cabo encuentros con otras experiencias y hacer comparaciones que son siempre un aporte relevante dentro de la producción de saberes, porque recogen diferentes miradas que enriquecen la sistematización y benefician a sus actores implícitos y explícitos.

Por esta razón, la finalidad de sistematizar la experiencia de los docentes en su práctica pedagógica, durante la pandemia mundial, tiene como propósito conocer las nociones que se han ido creando alrededor de este rol en los diferentes contextos; recogiendo la voz propia de quien ejerce y cumple estas demandas y exigencias, desde su proceso de aprendizaje, la perspectiva del papel que desempeña día a día, como una oportunidad para conocer lo que realmente piensan las personas que participan en la práctica para construir pensamiento colectivo e institucional. 
Congreso Internacional de Gestión Educativa, 06 y 07 de noviembre de 2020.

Pontificia Universidad Católica del Perú

\section{Referencias bibliográficas}

Ainscow. M. (2001) Desarrollo de escuelas inclusivas. Madrid: Nancea.

Black-Hawhins, K.; Florian, L. Y Rouse, M. (2007) Achivement and Inclusion in Schools. Londres: Routledge.

Escallón, I.E, Richler, D, Porter, G, (2013), GUÍA DE INDICADORES DE PRÁCTICAS PEDAGÓGICAS QUE FAVORECEN LA ATENCIÓN A LA DIVERSIDAD, Bogotá Colombia, Fundación Compartir.

Cassasus, J. (2000). Problemas de la gestión educativa en América Latina (la tensión entre los paradigmas de tipo A y el tipo B). Unesco.

Díaz-Barriga, Ángel Plá, Sebastián Cruz Flores de la, Gabriela Aguilar Nery, Jesús Ducoing Watty, Patricia Barrón Tirado, María Concepción Alcántara Santuario, Armando. Educación y pandemia. Una visión académica. México: UNAM, 2020 .

Gimeno, J. (1995) Diversos y también desiguales. Qué hacer en educación en Kiriki, n. 38 , pp. 19-25.

Lopes, M. (2018). ¿Qué hay entre las políticas de inclusión y las prácticas pedagógicas en la escuela? Sudamérica: Revista de Ciencias Sociales, 0(9), 73-94.

Sander. B. (2002) Nuevas Tendencias en la Gestión Educativa: democracia y calidad. En <http//www.iacd.oas.org/la\%20Educa\%20123-125/sand.htm>

Soto Calderón, Ronald (2003). La inclusión educativa: Una tarea que le compete a toda una sociedad . Revista Electrónica "Actualidades Investigativas en Educación", 3(1),0.[fecha de Consulta 12 de Mayo de 2020]. ISSN: . Disponible en: https://www.redalyc.org/articulo.oa?id=447/44730104

Skliar, C. (2005). Poner en tela de juicio la normalidad, no la anormalidad. Políticas y falta de políticas en relación con las diferencias en educación. Revista Educación y Pedagogía, 17(41), 10-22. Recuperado el 19 de Junio de 2020, de https://dialnet.unirioja.es/descarga/articulo/1419230.pdf

Yarza de los Rios, A. (2008). Formación del profesorado en educación especial, inclusión educativa y dispositivos de formación en Colombia. Revista Colombiana De Educación, (54), 74.93. https://doi.org/10.17227/01203916.54rce74.93 